Publ. Mat. 64 (2020), 183-194

DOI: 10.5565 /PUBLMAT6412008

\title{
GROUPS WITH NO PROPER CONTRANORMAL SUBGROUPS
}

\author{
B. A. F. Wehrfritz
}

\begin{abstract}
We consider which groups $G$ are nilpotent if they have a nilpotent normal subgroup $N$ with $G / N$ a restricted soluble group and if $G$ is the only contranormal subgroup of $G$. This supplements Kurdachenko, Otal, and Subbotin work of 2009, where they consider the corresponding question but with $G / N$ nilpotent and $N$ a restricted soluble normal subgroup.
\end{abstract}

2010 Mathematics Subject Classification: 20F16, 20F18, 20 F19.

Key words: soluble group, nilpotent group, contranormal subgroup.

Let $H$ be a subgroup of a group $G$. Following Rose [8] we say $H$ is contranormal in $G$ if $H^{G}=\left\langle H^{g}: g \in G\right\rangle=G$. Further, we say $G$ is Rose-nilpotent if $G$ is the only contranormal subgroup of $G$. Clearly, as Rose undoubtedly knew, finite Rose-nilpotent groups are nilpotent and all nilpotent groups are Rose-nilpotent. (John Rose was a good algebraist who died far too young. I think his name is worth preserving.)

Kurdachenko, Otal, and Subbotin in [4] make a serious study of what I have termed Rose-nilpotence. In many ways their most striking conclusion is their Theorem A; namely, that if $N$ is a soluble-by-finite normal subgroup of the Rose-nilpotent $G$ such that $G / N$ is nilpotent and $N$ satisfies min- $G$ (the minimal condition on normal subgroups of $G$ contained in $N$ ), then $G$ is nilpotent. This raises the obvious question as to what happens if $N$ is nilpotent and $G / N$ is soluble-by-finite with min- $G$.

Restrictions are needed. Heineken and Mohamed in [2] construct an extension $G$ of a countable elementary abelian $p$-group by a Prüfer $p$-group such that the centre of $G$ is $\langle 1\rangle$ and every subgroup of $G$ is subnormal in $G$. Thus, this group is not nilpotent but is Rose-nilpotent. Clearly $G$ here is periodic, metabelian, locally nilpotent, and abelian-byChernikov. However, positive results exist, but we must at least avoid situations where $N$ contains a $G$-section that is an infinite elementary abelian $p$-group for primes $p$ for which $G / N$ contains an infinite $p$-subgroup. The following is our main conclusion. 
Theorem 1. Let $N$ be a nilpotent normal subgroup of the Rose-nilpotent group $G$ such that $G / N$ is soluble-by-finite with min-G. Under any one of the following three conditions $G$ is nilpotent.

(a) $N$ is a FAR-group.

(b) $G / N$ is finite.

(c) $N$ is a periodic $\pi^{\prime}$-group, where $\pi$ is a set of primes and $G / N$ is a $\pi$-group.

FAR-groups are defined and their basic properties developed in $[\mathbf{5}$, Chapter 5]. Thus, the hypotheses on $N$ in (a) are that $N$ satisfies $\min -p$, the minimal condition on $p$-subgroups, for every prime $p$ and $N$ has finite Hirsch number $h(N)$ (also called the torsion-free rank; it means that $N$ has a series of finite length with $h(N)$ factors infinite cyclic and the remaining factors all locally finite). Actually, we prove Theorem 1 under slightly weaker hypotheses that are more complicated to state; see Theorem 2 below.

In the theorem, (a) is our main result and (b) and (c) are important steps in the proof of (a), whose proof also uses [4]. Next, we consider linear Rose-nilpotent groups. There are some positive results, for example periodic such groups and finitely generated such groups are nilpotent, but much more, linear groups are a fertile source of examples of nonnilpotent Rose-nilpotent groups. In [4] there is an example of an uncountable metabelian Rose-nilpotent non-nilpotent group based on the 2 -adic integers. This group is isomorphic to a subgroup of $\mathrm{GL}(2, \mathbb{C})$. However, there are countable examples in all characteristics, torsion-free ones of finite (Prüfer) rank in characteristic 0 and extensions of elementary abelian groups by torsion-free abelian groups in positive characteristics.

Page 86 of [5] lists a tower of progressively weaker rank conditions for soluble groups. We pointed out above for metabelian groups of finite rank that Rose-nilpotence need not imply nilpotence and hence the same must apply to the two weaker conditions above finite rank (namely FAR and finite Hirsch number). For the remaining five conditions the results are all positive. The weakest condition below finite rank is the FATR condition, which can be defined as follows. A group $G$ is a finite extension of a soluble FATR group if and only if $h(G)$ is finite and $\tau(G)$, the unique maximal locally finite normal subgroup of $G$, is Chernikov. Then we prove the following.

Proposition 1. Let $G$ be a finite extension of a soluble FATR group. If $G$ is Rose-nilpotent, then $G$ is nilpotent. 
The following is the fundamental result from [4, Theorems A and B] and Corollaries A1, A2, B1, B2, and B3 in [4] being easy consequences of it.

Theorem 1.6 ([4]). Let $A$ be an abelian normal subgroup of the Rosenilpotent group $G$ such that $G / A$ is nilpotent. If A satisfies min- $G$, then $G$ is nilpotent.

Finally, in this paper we give an alternative and shorter proof of this important result. Working with descending central series instead of ascending central series produces direct simplifications as well as avoiding the need to use [4, Proposition 1.4 and Corollary 1.5].

\section{The proof of Theorem 1}

Note first that the hypotheses in the theorem are all preserved by homomorphisms. Also $G / N$ is nilpotent by [4] and then $\min -G$ yields that $G / N$ is actually Chernikov. Further, if $G / N^{\prime}$ is nilpotent, then $G$ is nilpotent by a theorem of P. Hall (e.g. [13, 1.23]). Thus, throughout the proof of the theorem we may assume that $N$ is abelian and $G / N$ is Chernikov.

Lemma 1. Let $A$ be an abelian normal subgroup of the Rose-nilpotent group $G$ such that $G / A$ is Chernikov with $G / C_{G}(A)$ finite, say of order n. Then $G$ is nilpotent.

Proof: Now $G / A$ is nilpotent (e.g. by [4]) and so, of course, is $G /[A, G]$. We break the proof into three steps.

Step 1: $[A, G]$ has no section isomorphic to a Prüfer $p$-group for any prime $p$.

For, suppose $X<Y \leq[A, G]$ with $Y / X$ a Prüfer group. There exists $Z<A$ with $A / X=Y / X \times Z / X$. Set $B=\bigcap_{g \in G} Z^{g}$. Then $A / B$ embeds into a direct product of $n$ Prüfer groups. Therefore, $P=G / B$ is Chernikov and also Rose-nilpotent. Hence $P$ is nilpotent and consequently its minimal subgroup $P^{\circ}$ of finite index is central in $P$ (e.g. [3, 1.F.1]). Then the kernel $Q=R / B$ of the transfer homomorphism of $P$ into $P^{\circ}$ is finite, normal, and satisfies $P=P^{\circ} Q$. Hence $P^{\prime} \leq Q$, so $Y \leq[A, G] \leq R$. But $B \leq Z$, so $Y \cap B \leq Y \cap Z=X$ and hence $|Y / X| \leq|Y B / B| \leq|R / B|=|Q|<\infty$. However, $Y / X$ is infinite. This contradiction completes the proof of Step 1.

Step 2: If $[A, G]$ is periodic, then $G$ is nilpotent. 
By Step 1 each primary component of $[A, G]$ is its own basic subgroup $([\mathbf{1}, 32.3])$, is a direct product of cyclic groups, and by Step 1 again has finite exponent. Let $\pi$ denote the (finite) set of prime divisors of $n$. Then $[A, G]=P \times Q$, where $P$ is a $\pi$-group and $Q$ is a $\pi^{\prime}$-group. If $x \in Q \backslash\langle 1\rangle$, there is a subgroup $B$ of $[A, G]$ containing $P$ but not $x$ and of finite index in $P Q$. Replacing $B$ by $\bigcap_{G} B^{g}$ if necessary, we may choose $B$ normal in $G$ (of course $P$ and $Q$ are normal in $G$ ). Now $G / B$ is finite-by-nilpotent, so $G / B$ is nilpotent by [4]. But $P Q / B$ is a $\pi^{\prime}$-group and $G / C_{G}(P Q)$ is a finite $\pi$-group (of order dividing $n$ ). Therefore, $[P Q, G] \leq B$. Thus, $\left[A,{ }_{2} G\right] \leq \bigcap_{B} B=P$.

If instead we choose $x \in P \backslash\langle 1\rangle$ there exists a normal subgroup $B$ of $G$ in $P Q$ containing $Q$ but not $x$ with $B$ of finite index in $P Q$ and with $G / B$ nilpotent. Now $P$ has finite exponent, $e$ say, so if $\mathbf{g}$ denotes the augmentation ideal of $G / C_{G}(A)$ in its group $\operatorname{ring}$ over $\mathbf{Z} / \mathbf{Z} e$, then $\mathbf{g}^{m}=\mathbf{g}^{m+1}$ for some $m \leq e n$. Further, $\left[P Q,{ }_{j} G\right]$ in additive notation equals $(P Q) \mathbf{g}^{j}$ for each $j \geq 0$. Hence $\left[P Q,{ }_{m} G\right] \leq B$ and $\left[A,{ }_{m+1} G\right] \leq$ $\bigcap_{B} B=Q$. Consequently $[A, m+1 G] \leq P \cap Q=\langle 1\rangle$, and therefore $G$ is nilpotent, completing the proof of Step 2 .

Step 3: The completion of the proof of the lemma.

Now $[A, G]$ does not contain by Step 1 any free abelian subgroup of infinite rank. Hence $[A, G]$ contains a free abelian $B$ of finite rank, $r \geq$ 0 say, such that $[A, G] / B$ is periodic. Replacing $B$ by $\bigcap_{G} B^{g}$ as usual we may choose $B$ normal in $G$. If $p$ is any prime, then $[A, G] / B^{p}$ is periodic and hence $G / B^{p}$ is nilpotent by Step 2. Consequently $\left[B,{ }_{r} G\right] \leq B^{p}$ and so $\left[B,{ }_{r} G\right] \leq \bigcap^{p} B^{p}=\langle 1\rangle$. Clearly $G / B$ is nilpotent. Therefore, $G$ is nilpotent. The proof is now complete.

Lemma 2. Let $A$ be an abelian normal subgroup of the Rose-nilpotent group $G$. If for some set $\pi$ of primes $G / A$ is a Chernikov $\pi$-group and $A$ is a periodic $\pi^{\prime}$-group, then $G$ is nilpotent.

Proof: Now $G / A$ is nilpotent (e.g. by [4]) and $G$ is locally finite satisfying min- $\pi$, the minimal condition on $\pi$-subgroups. If $p \in \pi$, then $G$ contains a Sylow $p$-subgroup $P$; that is, a maximal $p$-subgroup $P$ of $G$ containing isomorphic copies of every $p$-subgroup of $G$ (see $[\mathbf{3}, 3.7]$ ). Let $P^{\circ}$ denote the minimal subgroup of $P$ of finite index. Then $P \cong P A / A$ is nilpotent, so $P^{\circ}$ is central in $P$ (see $[3,1$. F.1]). For each positive integer $m$ set $P_{m}=\left\{x \in P^{\circ}: x^{m}=1\right\} \leq P$ and let $K$ denote the kernel of the transfer homomorphism of $P$ into $P^{\mathrm{o}}$. Then $K P^{\mathrm{o}}=P$ and the set of all $K P_{m}$, as $P$ ranges over all the Sylow $p$-subgroups of $G$ but for fixed $m$, is a characteristic conjugacy class of subgroups of $G$ (see [3, 3.9 and 3.10]). 
Set $H=N_{G}\left(K P_{m}\right)$. If $g \in G$, then $P$ and $P^{g}$ are Sylow $p$-subgroups of $L=\left\langle H, H^{g}\right\rangle$ and hence by $[3,3.10]$ there exists $h$ in $L$ such that $\left(K P_{m}\right)^{g}=\left(K P_{m}\right)^{h}$. Thus, $g h^{-1} \in H$ and $g \in L$. Hence $H$ is abnormal in $G$ and $H^{G}=G$. But $G$ is Rose-nilpotent. Therefore $H=G$, the subgroups $K P_{m}$ are normal in $G$ for all $m$, and so $P$ is normal in $G$. Then $[A, P] \leq A \cap P=\langle 1\rangle$, since $A$ is a $\pi^{\prime}$-group and $P$ is a $\pi$-group and this is for every $p$ in $\pi$. Therefore, $C_{G}(A)=G$ by $[\mathbf{3}, 3.13]$. But $G / A$ is nilpotent. Consequently $G$ is nilpotent.

Lemma 3. Let $A$ be an abelian normal subgroup of the Rose-nilpotent group $G$ such that $G / A$ is a Chernikov $\pi$-group for some finite set $\pi$ of primes. Suppose $A$ has finite Hirsch number (= torsion-free rank) and satisfies min-p for each $p$ in $\pi$. Then $G$ is nilpotent.

Proof: Set $T=O_{\pi^{\prime}}(A)\left(=O_{\pi^{\prime}}(G)\right.$ note $)$. Suppose first that $O_{\pi}(A)=$ $\langle 1\rangle=T$. Then $A$ is torsion-free of finite rank, $r$ say. Hence $G / C_{G}(A)$ is isomorphic to a periodic subgroup of $\mathrm{GL}(r, \mathbf{Q})$ and consequently is finite (e.g. $[\mathbf{9}, 9.33])$. Therefore, $G$ is nilpotent by Lemma 1 . Then the image of $G / C_{G}(A)$ in $\operatorname{GL}(r, \mathbf{Q})$ is unipotent and hence torsion-free as well as finite. Consequently $[A, G]=\langle 1\rangle$.

Returning to the general case $O_{\pi}(A)$ is Chernikov, so $O_{\pi}(G)$ is too. If $G / O_{\pi}(G)$ is nilpotent, since $O_{\pi}(G)$ is Chernikov, then $G$ too is nilpotent by [4]. Therefore, assume from now on that $O_{\pi}(G)=\langle 1\rangle$. The case where $T=\langle 1\rangle$ is covered by the above, so assume that $T \neq\langle 1\rangle$. Now clearly $O_{\pi}(A / T)=O_{\pi}(A) T / T=\langle 1\rangle=O_{\pi}(A / T)$. Hence by the first case above we have $G / T$ nilpotent with $[A, G] \leq T$.

Now $[A, G] \leq T \leq A \leq C_{G}(A) \leq Z$, where $Z / C_{G}(A)$ is the centre of $G / C_{G}(A)$. Let $z \in Z$. Then $\phi: a \mapsto[a, z]$ is a $G$-homomorphism of $A$ into $T$ and $C_{A}(z)=\operatorname{ker} \phi$. Hence $C_{A}(z)$ is normal in $G$ and $A / C_{A}(z)$ is a $\pi^{\prime}$-group. Then $G / C_{A}(z)$ is nilpotent by Lemma 2 , for $G / A$ is a Chernikov $\pi$-group. Therefore, $[A, G] \leq C_{A}(z)$ and, consequently, $[A, z]=$ $\operatorname{Im} \phi$ is centralized by $G$. This is for all $z$ in $Z$ and hence $[A, Z, G]=\langle 1\rangle$.

Clearly $Z \leq C_{G}(A /[A, Z])$, so a simple induction applied to $T /[A, Z] \leq$ $A /[A, Z] \leq G /[A, Z]$ yields that $\left[A,{ }_{c} G\right]=\langle 1\rangle$ for some $c$ (e.g. $c$ the nilpotency class of $G / A$ ) and hence $G$ is nilpotent as required. (Actually here $T$ is a $\pi^{\prime}$-group, $A$ centralizes $T$, and $G / A$ is a $\pi$-group, so $[T, G]=$ $\langle 1\rangle$.)

Theorem 2 below and hence also Theorem 1 follow at once from P. Hall's theorem $([\mathbf{1 3}, 1.23])$ and Lemmas 1,2 , and 3 . 
Theorem 2. Let $N$ be a nilpotent normal subgroup of the Rose-nilpotent group $G$ such that $G / N$ is soluble-by-finite with min-G. Under any one of the following three conditions $G$ is nilpotent.

(a) $N / N^{\prime}$ satisfies min-p for every prime $p$ for which $G / N$ contains an element of order $p$ and $N / N^{\prime}$ has finite torsion-free rank.

(b) $\left(G: C_{G}\left(N / N^{\prime}\right)\right)$ is finite.

(c) $N / N^{\prime}$ is a periodic $\pi^{\prime}$-group, where $\pi$ is a set of primes and $G / N$ is a $\pi$-group.

The set of primes $p$ in (a) is always finite, given the other hypotheses on $G / N$. Actually (a) in Theorem 2 is not a lot stronger than (a) in Theorem 1, and (c) in Theorem 2 is no stronger than (c) in Theorem 1. These follow from the easy facts that for a nilpotent group $N$, if $N / N^{\prime}$ is a FAR-group, then so is $N$ and if $N / N^{\prime}$ is a $\pi^{\prime}$-group, then so is $N$; use [13, Sublemma (b) on p. 10] or $[6,2.26]$.

\section{Linear groups}

If $\mathbf{X}$ is a class of groups such that $\mathbf{X}$-groups all of whose finite images are nilpotent are themselves nilpotent, then clearly Rose-nilpotent $\mathbf{X}$-groups are nilpotent. Below we give examples of such classes $\mathbf{X}$.

(a) Finitely generated linear groups.

(b) Subgroups of the groups $\operatorname{GL}(n, R)$, for any positive integer $n$ and any finitely generated (commutative) integral domain $R$. See $[\mathbf{9}$, 4.16].

(c) Finite extensions of torsion-free soluble minimax groups. This follows from (b) since for each such group there exist positive integers $m$ and $n$ such that $G$ embeds into $\operatorname{GL}(n, \mathbf{Z}[1 / m])$. See 1.3 of $[\mathbf{1 0}]$.

(d) Finitely generated subgroups of the groups $\operatorname{Aut}_{R} M$ for $M$ any Noetherian module over any commutative ring $R$. Apply 6.1 of [11] and (a) above.

(e) Finitely generated subgroups of the groups $\operatorname{Aut}_{R} M$ for $M$ any Artinian module over any commutative $\operatorname{ring} R$ with $R / \operatorname{Ann}_{R} M$ is Noetherian. See 3.2 of [12].

(f) Finitely generated hyper (abelian or finite) groups. See [6, 10.51].

Proposition 2. Periodic Rose-nilpotent linear groups are nilpotent.

Proof: Let $G$ be a periodic Rose-nilpotent subgroup of $\operatorname{GL}(n, F), n$ an integer, $F$ some field. Let $p$ be any prime and $P$ a maximal $p$-subgroup of $G$. Set $H=N_{G}(P)$. If $H<G$, then $N=H^{G}<G$ by Rose-nilpotence. 
By $[\mathbf{9}, 9.10]$ (the analogue of Sylow's theorem) and the Frattini argument $G=H N=N$. Hence $H=G$ and so $G=\times_{p}$ prime $P$. If $p=\operatorname{char} F$, then $P$ is nilpotent (of class less than $n$ ). If $p \neq \operatorname{char} F$ and $p>n$, then $P$ is abelian. If $p \neq \operatorname{char} F$ and $p \leq n$, then $P$ is Chernikov. (See $[\mathbf{9}, 9.1]$ for these results.) However, $P$ as an image of $G$ is Rose-nilpotent, so in the final case here $P$ is at least nilpotent ([4]). Therefore, $G$ is nilpotent. (Apart from the trivial cases where $n=1$ or $n=2=\operatorname{char} F$, there is no general bound on the nilpotency class of $G$ in terms of $n$ and char $F$; see $[\mathbf{9}, 8.3]$.)

This proposition, $[\mathbf{1 1}, 6.1]$, and $[\mathbf{1 2}, 3.2]$ immediately yield the following.

Corollary. Let $G$ be a periodic Rose-nilpotent subgroup of $\mathrm{Aut}_{R} M$, where $R$ is a commutative ring and $M$ is either a Noetherian $R$-module or an Artinian $R$-module with $R / A_{R} M$ Noetherian. Then $G$ is nilpotent.

We now consider linear counter examples. As pointed out in [4] the infinite locally dihedral 2-group $G$ is hypercentral but not Rose-nilpotent. Clearly this $G$ has a faithful linear representation of degree 2 over any large enough field of characteristic not 2 .

Lemma 4. Let $G=U A$ be the split extension of the abelian group $A$ by the abelian group $U$. Then $G$ is Rose-nilpotent if and only if for all $X<$ $A$, a normal subgroup of $G$, we have $X[A, U]<A$.

Proof: Suppose $G$ is Rose-nilpotent and $X<A$ is a normal subgroup of $G$. Set $H=U X<G$. Now $H^{G} \geq H[A, H] \geq U X[A, U]$. Thus, $H^{G} \cap A \geq X[A, U]$. If $A \leq H^{G}$, then $H^{G}=G$, so by Rose-nilpotence $H^{G} \cap A<A$ and hence $X[A, U]<A$.

Now assume $G$ is not Rose-nilpotent. Then there exists $H<G$ with $H^{G}=G$. Since $U$ is abelian, $H^{G} \leq H A$, so $H A=G$. Therefore, $X=H \cap A$ is normal in $G$ and $H<G$, so $X<A$. Finally, $A \leq G=$ $H^{G}=H[A, H]=H[A, G]=H[A, U]$, so $A=X[A, U]$.

Corollary. Let $R$ be a commutative local ring with maximal ideal $\mathbf{m}$. Suppose $\mathbf{m}$ is not nilpotent and $R / \mathbf{m}=G F(p)$ for some prime $p$. Let $A$ denote the additive group of $R$ and $U=1+\mathbf{m}$, a subgroup of the group of units of $R$. Then the split extension $G$ of $A$ by $U$ is Rose-nilpotent but not nilpotent. Also $G$ is isomorphic to a subgroup of $\operatorname{Tr}(2, R)$. If $R$ is an integral domain, then $G$ is not even locally nilpotent.

Proof: Clearly $\mathbf{Z} 1_{R}[U]=R$. Thus, if $X<A$ is a normal subgroup of $G$, then $X$ is an ideal of $R$. Thus, $X \leq \mathbf{m}$. Also $[A, U]=R \mathbf{m}=\mathbf{m}$, so 
$X+[A, U] \leq \mathbf{m}<A$. Hence $G$ is Rose-nilpotent by Lemma 4. Also $\left[A,{ }_{c} U\right]=\mathbf{m}^{c}$ for all $c \geq 1$ and by hypothesis $\mathbf{m}^{c} \neq\{0\}$. Therefore, $G$ is not nilpotent. Finally, if $a \in A$ and $1+x \in U$ with $\langle a, 1+x\rangle$ nilpotent, then $\left[a,{ }_{c} 1+x\right]=1$ for some $c$; that is, as elements of $R$ we have $a x^{c}=0$. If $R$ is a domain, then either $a=0$ or $1+x=1$, so $G$ cannot be locally nilpotent.

We now consider special cases of this corollary.

(1) For any prime $p$ let $R=\mathbf{Z}[1 / q$ : primes $q \neq p]$. Then $R$ satisfies the corollary and $G$ is Rose-nilpotent, not locally nilpotent, metabelian, countable, torsion-free ((torsion-free)-by-(of index 2) if $p=2)$, and isomorphic to a subgroup of $\operatorname{Tr}(2, R) \leq \operatorname{Tr}(2, \mathbf{Q}) \leq$ $\mathrm{GL}(2, \mathbf{Q})$.

(2) For any prime $p$ set $F=G F(p)$ and let $x$ be an indeterminant over $F$. Let $R$ denote the local ring of $F[x]$ over its maximal ideal $x F[x]$. Again $R$ satisfies the corollary. Here $G$ is Rose-nilpotent, not locally nilpotent, metabelian, countable, an (elementary abelian $p$-group)-by-(torsion-free abelian), and isomorphic to a subgroup of $\operatorname{Tr}(2, R) \leq \operatorname{Tr}(2, F(x)) \leq \mathrm{GL}(2, F(x))$.

(3) If $p$ is any prime the ring $R$ of $p$-adic integers satisfies the corollary. Here $G$ is uncountable and $G$ is Rose-nilpotent, not locally nilpotent, metabelian, torsion-free ((torsion-free)-by-(of index 2) if $p=2)$, and isomorphic to subgroups of $\operatorname{Tr}(2, R)$ and $\operatorname{Tr}(2, \mathbf{C})$. If $p=2$, then $G$ here is almost the same as the example given on p. 235 of $[\mathbf{4}]$.

Lemma 4 can be used to exhibit other interesting, but now non-linear examples. For each prime $p$ let $A_{p}$ be a cyclic group of order $p^{p}$. Then $A_{p}$ has an automorphism $b_{p}$ of order $p^{p-1}$ and $G_{p}=\left\langle b_{p}\right\rangle A_{p}$ is nilpotent of class $p$. Set $A=\times_{p} A_{p}$ and $G=\times_{p} G_{p}$. If $X$ is a proper subgroup of $A$, then $X=\times_{p}\left(X \cap A_{p}\right)$ and there is at least one prime $q$ such that $X \leq Y=\left(\times_{p \neq q} A_{p}\right) \times\left(A_{q}\right)^{q}$. Also $[A, G]=\times_{p}\left(A_{p}\right)^{p} \leq Y$ and $Y$ is normal in $G$. Therefore, $G$ is Rose-nilpotent by Lemma 4. Thus:

(4) The group $G$ is metabelian, hypercentral, periodic, of rank 2, and Rose-nilpotent but not nilpotent.

As a variation of (4) let $\langle b\rangle$ be an infinite cyclic group acting on $A$ by $b$ acting as $b_{p}$ on each $A_{p}$. Now set $G=\langle b\rangle A$. Again $[A, G]=\times_{p}\left(A_{p}\right)^{p}$ and the argument above yields that $G$ is Rose-nilpotent. Thus:

(5) The group $G$ is (locally cyclic)-by-(infinite cyclic), hypercentral, of rank 2, and Rose-nilpotent but not nilpotent. (Incidentally this also shows that we cannot replace $\min -G$ by $\max -G$ in Theorems 1 and 2.) 


\section{Proof of Proposition 1}

Note that a minor complication of working with the class of FATR groups is that it is not quotient-closed (just consider $\mathbf{Q} / \mathbf{Z}$ ).

We induct on $h(G)$; if $h(G)=0$, then $G$ is Chernikov and nilpotent. If $G / \tau(G)$ is nilpotent, then so is $G$ by $[\mathbf{4}$, Theorem A], so assume $\tau(G)=$ $\langle 1\rangle$. Then $G$ has a torsion-free nilpotent normal subgroup $N$ such that $G / N$ is finitely generated and abelian-by-finite, see [5, 5.2.2 and 5.2.3]. Then $G / N$ is nilpotent and $G$ is soluble. In particular if $N=\langle 1\rangle$, then $G$ is nilpotent, so assume $N \neq\langle 1\rangle$. Now the centre $A$ of $N$ is a torsionfree abelian normal subgroup of $G$ of finite rank $r \geq 1, N / A$ is torsionfree $([6,2.25])$, and $\tau(G / A)$ is finite. By induction $G / A$ is nilpotent.

Now $G=T N$ for some finitely generated subgroup $T$ of $G$ and $T /(T \cap$ $A$ ) is nilpotent. There exists a free abelian subgroup $B$ of $A$ of rank $r$ with $A / B$ periodic. Since $A$ is central in $N$ and $G=T N$ we have that $T \cap A$ is normal in $G$ and $B^{G}=B^{T}$. Set $D=B^{G}(T \cap A) \leq A$ and $H=T B^{G}=T D$. Then $H$ is a finitely generated (and soluble) subgroup of $G$.

Let $K$ be a normal subgroup of $H$ of finite index. Then $K \cap D$ is a normal subgroup of $G$ (again using that $A \geq D$ is central in $N$ and $G=$ $T N)$ and $A /(K \cap D)$ is periodic abelian of finite rank. As such, each of the primary components of $A /(K \cap D)$ is Chernikov. Thus, there exists a normal subgroup $C \geq K \cap D$ of $G$ in $A$ such that $A / C$ is Chernikov and $C \cap D=K \cap D$. By [4, Theorem A] again $G / C$ is nilpotent. Thus, for some integer $k$ we have $\left[D,{ }_{k} G\right] \leq K$. But $H / D$ is an image of $T /(T \cap A)$, which is nilpotent. Therefore, $H / K$ is nilpotent. This for all such $K$. Consequently $H$ is nilpotent by $[\mathbf{6}, 10.51]$.

From the above it follows that $\left[D,{ }_{h} H\right]=\langle 1\rangle$ for some integer $h$ and $\left[D,{ }_{h} H\right]=\left[D,{ }_{h} G\right]$ since $D \leq A$ and $G=H N$. But $A \geq D \geq B \neq$ $\langle 1\rangle$. Therefore, $C_{A}(G) \neq\langle 1\rangle$. Also $A / C_{A}(G)$ is torsion-free (since $A$ is torsion-free and $\left[a^{n}, g\right]=[a, g]^{n}$ for all $a$ in $A, g$ in $G$, and integers $n$ ), so $\tau\left(G / C_{A}(G)\right)$ is finite. Induction on $h(G)$ yields that $G / C_{A}(G)$ is nilpotent. Consequently so is $G$. The proof is complete.

We now return briefly to linear groups.

Corollary. For any integer n, let $G$ be a Rose-nilpotent soluble-by-finite subgroup of $\mathrm{GL}(n, \mathbf{Q})$ that is unipotent-by-(finitely generated). Then $G$ is nilpotent.

By (1) above, Rose-nilpotent metabelian subgroups of GL(2, Q) need not be nilpotent, so we need the extra hypothesis. 
Proof: Unipotent subgroups of $\mathrm{GL}(n, \mathbf{Q})$ are torsion-free nilpotent of finite rank and $G$ is unipotent-by-abelian-by-finite. Thus, $G$ satisfies the hypotheses of Proposition 1 and hence is nilpotent. (Actually if $G$ is as in Proposition 1, then $G / \tau(G)$ embeds into some $\operatorname{GL}(n, \mathbf{Q})$, see [10], so the proposition and its corollary are more or less equivalent.)

\section{A proof of $[4$, Theorem 1.6]}

If $n \geq 0$ is an integer and if $x$ is an element of a group $G$, set

$$
E_{G, n}(x)=\left\{g \in G:\left[g,{ }_{n} x\right]=1\right\} .
$$

Obviously $E_{G, 0}(x)=\langle 1\rangle$ and $x \in E_{G, n}(x)$ for all $n \geq 1$. Given $n$ and $x$, the set $E_{G, n}(x)$ need not be a subgroup of $G$, but if $[G, x]$ is nilpotent of class $c$ there always exists an integer $m=m(n, c)$ such that $\left\langle E_{G, n} n(x)\right\rangle \subseteq E_{G, m}(x)$. This follows from a couple of elementary commutator formulae (see $[\mathbf{7}$, Lemma 4]) and a simple induction (see $[\mathbf{4}$, Lemma 1.3], cf. [7, Corollary $\left.3^{*}\right]$ ).

We are given an abelian normal subgroup $A$ of the Rose-nilpotent group $G$ such that $A$ satisfies min- $G$ and $G / A$ is nilpotent of class $c$. We have to prove that $G$ is nilpotent.

Suppose $G$ is not nilpotent. By min- $G$ we may assume that $A=[A, G]$. Since $G$ is not nilpotent but $G / A$ is nilpotent, so $A \leq C_{G}(A)<G$ and we may pick an $x$ in $G \backslash C_{G}(A)$ with $[G, x] \leq C_{G}(A)$. Since $x$ is central in $G$ modulo $C_{G}(A)$, each $\left[A,{ }_{j} x\right]$ is normal in $G$ for $j \geq 0$. Hence $\left[A,{ }_{s} x\right]=\left[A,{ }_{s+1} x\right]=B$ say, for some minimal $s \geq 1$.

Let $g \in G$. Then $\left[g,{ }_{c+s} x\right] \in B=[B, x]$ and hence there exists $b$ in $B$ with $\left[b,{ }_{c+s} x\right]=\left[g,{ }_{c+s} x\right]$. Since $[G, x]$ and $A$ commute this implies that

$$
\left[g b^{-1},{ }_{c+s} x\right]=\left[g,{ }_{c+s} x\right]\left[b,{ }_{c+s} x\right]^{-1}=1 .
$$

Hence $E_{G, c+s}(x) B=G$. Set $L=\left\langle E_{G, c+s}(x)\right\rangle$, so $L B=G$. Now $[G, x] \leq$ $C_{G}(A)$, which is nilpotent (of class at most $c+1$ ). Thus, $L \subseteq E_{G, m}(x)$ for some $m$, see above. Also $L^{G} \geq L[A, L]=L[A, G]=L A=G$. Since $G$ is Rose-nilpotent, this implies that $L=G$. But then $L \geq B$, so $\left[B,{ }_{m} x\right]=$ $\langle 1\rangle$. Hence if $B \neq\langle 1\rangle$, we have $B>[B, x]=B$. Consequently $B=\langle 1\rangle$. Therefore, $[A, s x]=\langle 1\rangle$. Since $x \notin C_{G}(A)$ we have $s \geq 2$.

Set $D=\left[A,{ }_{s-1} x\right]>\langle 1\rangle$. Now, thinking of $A$ as a $G$-module, in additive notation $D=A(x-1)^{s-1}$ and $(x-1)^{s-1}$ defines a $G$-homomorphism $\phi$ of $A$ onto $D$, say with kernel $E$. Then $E$ is a normal subgroup of $G$ with $[A, x]=A(x-1) \leq E<A$, using $A / E \cong D \neq\langle 1\rangle$. Repeat the above but with $A$ and $G$ replaced by $A / E$ and $G / E$. Thus, we choose $y$ in $G \backslash C_{G}(A / E)$ and an integer $t$ with $[G, y] \leq C_{G}(A / E)$, 
$\left[A,{ }_{t} y\right] \leq E,\left[A,{ }_{t-1} y\right]$ not contained in $E$ and $t \geq 2$. Thus, using the $G$-homomorphism $\phi$ we now have

$$
D>\left[D,{ }_{t-1} y\right]>\langle 1\rangle \text {; that is, }\left[A,{ }_{s-1} x\right]>\left[A,{ }_{s-1} x,{ }_{t-1} y\right]>\langle 1\rangle .
$$

Note that $(x-1)^{s-1}(y-1)^{t-1}\left(\equiv(y-1)^{t-1}(x-1)^{s-1}\right.$ modulo $\operatorname{Ann}_{Z G}(A)$ does determine a $G$-homomorphism $\psi$ of $A$ into itself, indeed into $D$.

Now apply this argument to $A$ and $G$ modulo $F=\operatorname{ker} \psi$. Then $A>F>E$ and we produce $z \in G \backslash C_{G}(A / F)$ with $[G, z] \leq C_{G}(A / F)$ and an integer $u \geq 2$ with $\left[A,{ }_{u} z\right] \leq \mathrm{F},\left[A,{ }_{u-1} z\right]$ not contained in $F$ and satisfying

$$
\left[A,{ }_{s-1} x\right]>\left[A,{ }_{s-1} x,{ }_{t-1} y\right]>\left[A,{ }_{s-1} x,_{t-1} y,{ }_{u-1} z\right]>\langle 1\rangle .
$$

In this way, by keep repeating the above argument, we can construct an infinite strictly descending series of normal subgroups of $G$ in $A$, contradicting the min- $G$ hypothesis. Therefore, $G$ is nilpotent.

Remark (Chernikov groups). We have repeatedly used above the fact that Chernikov Rose-nilpotent groups are nilpotent, justifying its use by quoting Theorem A of [4]. However, it is a much more elementary result than the theorems of [4]. Perhaps it is worthwhile recording a short elementary proof.

Thus, let $G$ be a Chernikov Rose-nilpotent group, $A$ its minimal subgroup of finite index, $n=(G: A), T$ a transversal of $A$ to $G, H=\langle T\rangle$, and $\gamma=\sum_{t \in T} t \in \mathbf{Z} G$. Suppose $G$ is not nilpotent, so $G$ is infinite and $r=\operatorname{total}-$ rank $A \geq 1$. We induct on $r$. Now $A \gamma$ is divisible and central in $G$. If $A \gamma \neq\langle 1\rangle$, then $G / A \gamma$ is nilpotent by induction, so $G$ is too. Hence assume $A \gamma=\langle 1\rangle$. If $a \in A$, then in additive notation $n a=\sum_{T} a(1-t)$. Thus, in multiplicative notation $[A, H] \geq A^{n}=A$. Hence $H^{G} \geq H[A, H] \geq H A=G$. But $G$ is infinite and $H$ is finite, so $H<G$. This contradicts Rose-nilpotence and completes the proof that $G$ is nilpotent.

\section{References}

[1] L. Fuchs, "Infinite Abelian Groups", Vol. I, Pure and Applied Mathematics 36, Academic Press, New York-London, 1970.

[2] H. Heineken and I. J. Mohamed, A group with trivial centre satisfying the normalizer condition, J. Algebra 10(3) (1968), 368-376. DOI : 10.1016/0021-8693 (68) $90086-0$.

[3] O. H. Kegel and B. A. F. Wehrfritz, "Locally Finite Groups", North-Holland Mathematical Library 3, North-Holland Publishing Co., Amsterdam-London; American Elsevier Publishing Co., Inc., New York, 1973. 
[4] L. A. Kurdachenko, J. Otal, and I. Ya. Subbotin, Criteria of nilpotency and influence of contranormal subgroups on the structure of infinite groups, Turkish J. Math. 33(3) (2009), 227-237. DOI : 10.3906/mat-0805-20.

[5] J. C. Lennox and D. J. S. Robinson, "The Theory of Infinite Soluble Groups", Oxford Mathematical Monographs, The Clarendon Press, Oxford University Press, Oxford, 2004. DOI : 10.1093/acprof : oso/9780198507284.001.0001.

[6] D. J. S. Robinson, "Finiteness Conditions and Generalized Soluble Groups", Parts 1 and 2, Ergebnisse der Mathematik und ihrer Grenzgebiete 62 and 63, Springer-Verlag, New York-Berlin, 1972. DOI : 10.1007/978-3-662-07241-7. DOI : 10.1007/978-3-662-11747-7.

[7] D. J. S. Robinson, Splitting theorems for infinite groups, in: "Symposia Mathematica, Vol. XVII" (Convegno sui Gruppi Infiniti, INDAM, Rome, 1973), Academic Press, London, 1976, pp. 441-470.

[8] J. S. Rose, Nilpotent subgroups of finite soluble groups, Math. Z. 106(2) (1968), 97-112. DOI : $10.1007 / \mathrm{BF} 01110717$.

[9] B. A. F. Wehrfritz, "Infinite Linear Groups. An Account of the Group-theoretic Properties of Infinite Groups of Matrices", Ergebnisse der Matematik und ihrer Grenzgebiete 76, Springer-Verlag, New York-Heidelberg, 1973. DOI : $10.1007 / 978-3-642-87081-1$.

[10] B. A. F. Wehrfritz, On the holomorphs of soluble groups of finite rank, $J$. Pure Appl. Algebra 4(1) (1974), 55-69. DOI : 10.1016/0022-4049(74) 90030-9.

[11] B. A. F. Wehrfritz, "Lectures Around Complete Local Rings", Queen Mary College Mathematics Notes, Queen Mary College, Department of Pure Mathematics, London, 1979.

[12] B. A. F. Wehrfritz, Artinian-finitary groups over commutative rings, Illinois J. Math. 47(1-2) (2003), 551-565. Special issue in honor of Reinhold Baer (1902-1979). DOI : 10.1215/ijm/1258488172.

[13] B. A. F. Wehrfritz, "Group and Ring Theoretic Properties of Polycyclic Groups", Algebra and Applications 10, Springer-Verlag London, Ltd., London, 2009. DOI : $10.1007 / 978-1-84882-941-1$.

School of Mathematical Sciences, Queen Mary University of London, London E1 4NS, England

E-mail address: b.a.f.wehrfritz@qmul.ac.uk

Primera versió rebuda el 8 de març de 2018, darrera versió rebuda el 25 d'abril de 2018. 